\title{
Electronic Thermal Expansion and the Mechanism of Coherent Acoustic Phonons Generation in Metals
}

\author{
S. Nie, H. Park, X. Wang, R. Clinite, and J. Cao \\ Physics Department / National High Magnetic Field Laboratory, Florida State University, 1800 E. Paul \\ Dirac Dr. Tallahassee, FL 32310, USA
}

Under thermal equilibrium conditions and at or above room temperature, the thermal expansion of metals is dominated by the lattice heating, largely due to that the lattice heat capacity is about two orders of magnitude larger than that of conduction electrons. In contrast, under the highly non-equilibrium conditions created by the fs optical excitation, the conduction electrons are transiently heated well above the lattice temperature thus can contribute significantly to the thermal expansion. By directly probing the associated thermal expansion dynamics in real time with femtosecond electron diffraction and performing a quantitative analysis using a classical harmonic oscillator model, we are able to separate the contribution of hot electrons from that of lattice heating and make quantitative and direct measurements of their contributions to driving coherent acoustic phonons. The results show that electronic thermal expansion contributes significantly to the generation of coherent acoustic phonons under such non-equilibrium conditions.

Ultrafast thermal expansion in free-standing polycrystalline Al films of 20 -nm thickness was initiated by ultrafast heating with $50-\mathrm{fs}$ near infra-red $(790 \mathrm{~nm})$ laser pulses. The associated structural dynamics were monitored by taking snapshots of diffraction patterns (see Fig. 1) at different delay times using transmission femtosecond electron diffraction.

Temporal evolutions of both coherent and thermal lattice motions are shown in Fig. $2 \& 3$. The coherent lattice vibration (coherent phonons), with a period of $\sim 6.0 \mathrm{ps}$, is characterized as a in-phase oscillation of all Bragg peaks around a new and expanded equilibrium lattice position, corresponding to an elevated lattice temperature of $\sim 25 \mathrm{~K}$. The lattice temperature displays an exponential temporal behavior with a time constant of $\sim 550$ fs.

We show that the phonon dynamics can be well-fitted with a damped classical harmonic oscillator model using a driving force that includes both electronic $\left(\sigma_{e}\right)$ and lattice $\left(\sigma_{l}\right)$ thermal stresses:

$$
\frac{d^{2} Q}{d t^{2}}+2 \beta \frac{d Q}{d t}+\omega_{0}^{2} Q=\sigma(t) \text { and } \sigma=\sigma_{l}+\sigma_{e}=C_{e} \gamma_{e} \delta T_{e}+C_{l} \gamma_{l} \delta T_{l}
$$

where $C_{e}$ and $C_{l}$ are heat capacities for electrons and phonons, $\gamma_{e}$ and $\gamma_{l}$ are the corresponding Grüneisen constants. Notably, fitting without $\sigma_{e}$ generates a significant phase lag of 22 degrees, indicating that the pressure of hot free electrons contributes significantly in driving the coherent acoustic phonons under the conditions when electrons and phonons are not thermalized [1].

In addition, using this transient heating method, we are also able to make accurate measurements of the electronic Grüneisen constant $\gamma_{e}$ of metals at room temperature using FED [2]. The obtained Grüneisen constant of $\mathrm{Al}, \gamma_{e}=1.4 \pm 0.3$, is in excellent agreement with that of 1.6 obtained in the low temperature measurement. This new approach opens the way to determining the $\gamma_{e}$ values in many magnetic materials, such as rare earth elements with low Curie temperatures, where the accurate measurement of $\gamma_{e}$ is not amenable with other traditional techniques.

\section{References}

[1] H. Park, et al., Phys. Rev. B, Rapid Commun. 72, 100301(R) (2005).

[2] S. Nie, et al., Phys. Rev. Lett. 96, 025901 (2006).

[3] This work was supported by the National Science Foundation under the Contract No. DMR-0305519. 

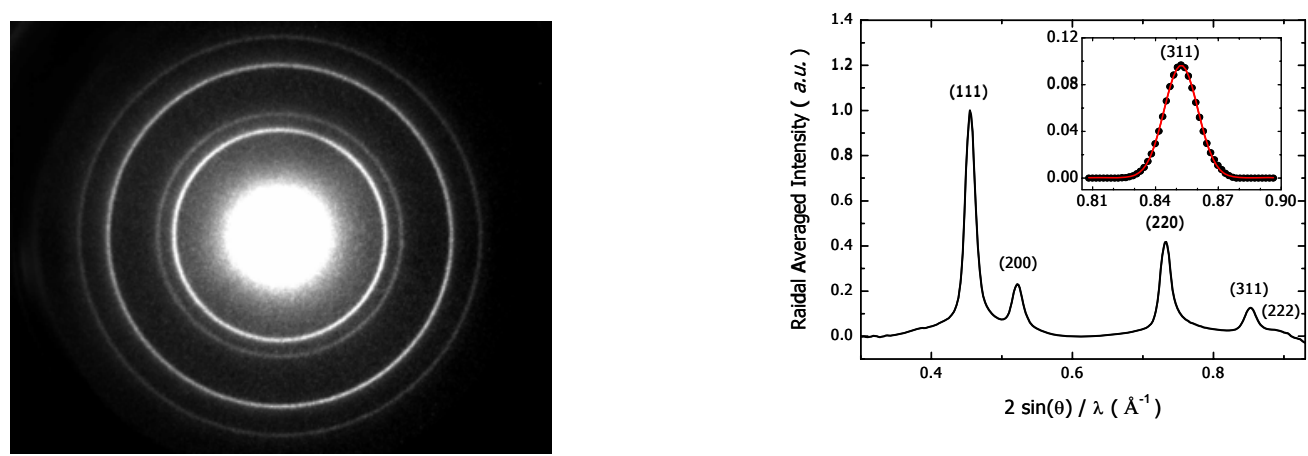

Fig. 1 Left Panel: Diffraction pattern of Al film of $20-\mathrm{nm}$ thickness. It was recorded with $\sim 2 \times 10^{7}$ electrons of $60 \mathrm{keV}$ beam energy $(\lambda=0.0487 \AA)$ at approximately $1.5 \mathrm{~mJ} / \mathrm{cm}^{2}$ laser excitation fluence. Right Panel: The corresponding radial averaged intensity curve. Inset: A typical fit of (311) Bragg peak to a Gaussian profile. The peak center position is determined to be $0.81780 \pm 0.00003\left(\AA^{-1}\right)$.
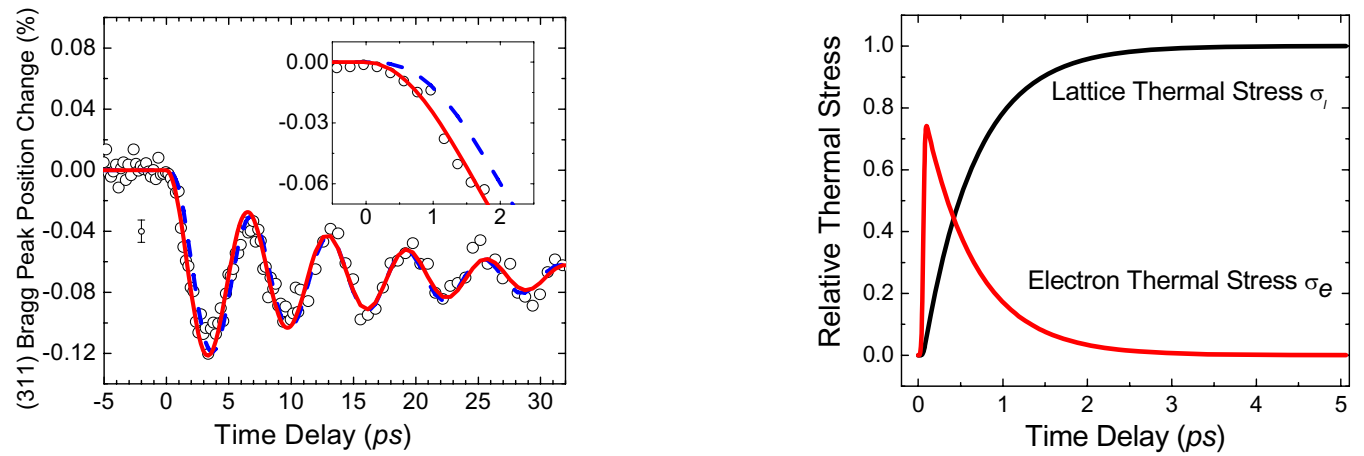

Fig. 2 Left panel: Temporal evolution of (311) Bragg peak positions. The solid line is the fit to the experimental data using harmonic oscillator model. The dashed curve is a fit excluding $\sigma_{e}$, which lags behind the data with a phase shift of approximately $22^{\circ}$. Inset: Detailed view of the above two fitting results in the range of -0.5 to 2.5 ps. Right panel: Temporal evolution of electron and lattice stress.
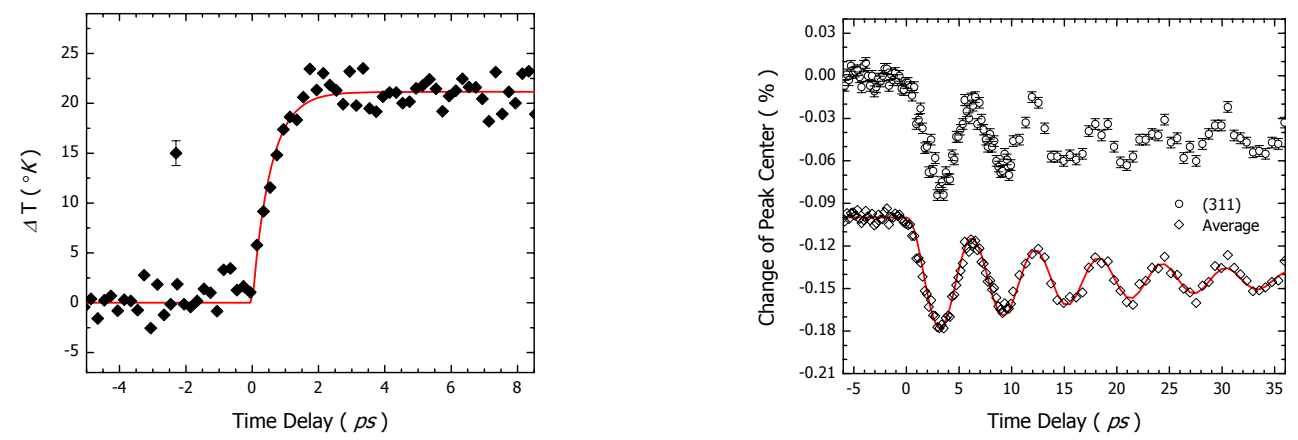

Fig. 3 Measurement of the electronic Grüneisen constant using FED. Left Panel: Temporal evolution of lattice temperature. The solid line is a fit to the data using an exponential function with a time constant $\tau_{e-p h}=550 \pm 80$ fs. Right Panel: Coherent lattice vibration and the data fit with the damped harmonic model. 